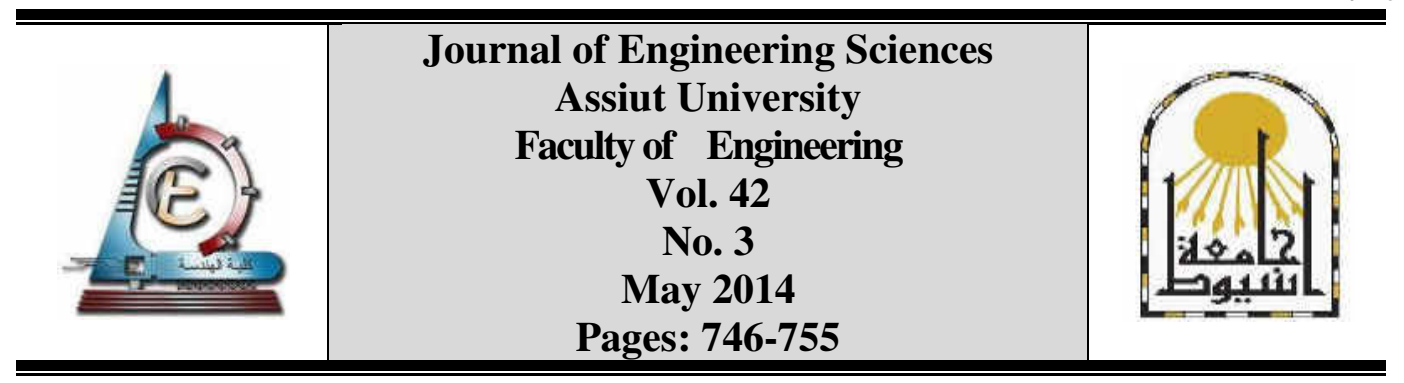

\title{
COMPRESSED MEASUREMENTS BASED CYCLOSTATIONARY DETECTOR FOR WIDEBAND COGNITIVE RADIOS
}

\author{
Mohammed Y. Abdelsadek *, Mohammed Farrag, and Taha A. Khalaf \\ Staff in Electrical Engineering Dept., Faculty of Engineering, Assiut University
}

Received 1 April 2014; revised 2 May; accepted 17 May 2014

\begin{abstract}
Cyclostationary feature detection is one of the most powerful spectrum sensing techniques used for cognitive radio (CR) systems. This is because of its robustness against noise uncertainties. However, this technique needs high sampling rates, which is limited by the state-of the-art analog to digital converters (ADCs), especially in wideband regime. Comressive sensing (CS) was used by many researchers for solving this problem via sub-Nyquist sampling rates. However CS solves the high sampling rate problem, but it does not reduce complexity considerably. This is because spectrum sensing is performed in three steps: sensing compressed measurements, then reconstructing the Nyquist rate signal, and finally performing cyclostationary detection (CD) on the reconstructed signal. In this paper we suggest performing $\mathrm{CD}$ directly on the compressed measurements skipping the reconstruction step which is the most complex step in CS. This can be realized by designing the sensing matrix with constraints different from those used in the conventional CS. Results show that performance is improved relative to applying CD on the Nyquist rate signal. This is in addition to reduction in receiver complexity resulting from reducing sampling rates. A detection probability of $78.7 \%$ can be achieved with only $7 \%$ of samples used by the conventional cyclostationary detection technique that achieves a detection probability of $32.7 \%$.
\end{abstract}

Keywords: Cognitive radio, Spectrum Sensing, Cyclostationary Detector, Compressive Sensing.

\section{Introduction}

The electromagnetic spectrum is a scarce resource, therefore it is coordinated by governmental agencies like the Federal Communications Commission (FCC) in USA and the National Telecommunication Regulatory Authority (NTRA) in Egypt. The FCC assigns spectrum to licensed holders on a long term basis for large geographical regions. However, a large portion of the assigned spectrum remains under utilized [1]. Cognitive radio (CR) enables users who have no spectrum licenses, known as secondary users (SUs), to use the

\footnotetext{
* Corresponding author.
}

Email address: myelsadek@yahoo.com 
temporarily unused spectrum of licensed or primary users (PUs). The main functions of CR's are: spectrum sensing, spectrum management, spectrum mobility, and spectrum sharing [2].

Spectrum sensing (SS) is the most important component for the establishment of CR. The fundamental task of SS is to detect the unused spectrum band and share it without harmful interference with the PUs [3]. There is a lot of spectrum sensing techniques, it can be mainly classified to primary transmitter detection and cooperative and collaborative detection [4]. Primary transmitter detection is based on the received signal by CR users, it includes detection techniques such as; matched filter detection [5], energy detection [6], and cyclostationary detection. In cooperative and collaborative detection [7], signals for spectrum opportunities are detected reliably by cooperating with other users.

SS is challenging due to the requirements set by the FCC on the sensing speed and accuracy [8]. This is particularly manifested in wide-band systems due to the high sampling rates required being at least equal to the Nyquist rate or twice the bandwidth of the sensed signal, which means complex and expensive hardware with high power consumption. Also, the timing requirements for rapid sensing may only allow for acquisition of a small number of samples, which may not provide sufficient statistics when conventional signal reconstruction methods are used.

Recently, compressive sensing (CS) is used in conjunction with CRs to solve the high sampling rates problem and some other hardware requirement challenges. CS is the technique of recovering the Nyquist rate samples of a signal from fewer samples sensed with sub-Nyquist rate [9], [10]. The sensing process can be performed using an Analog to Information Converter (AIC) to get a low dimensional data. Several matrices which represent the dimensionality reduction are used for sensing such as Gaussian, Bernoulli, and partial Hadamard and Fourier matrices. Whatever the used sensing matrix is, it should satisfy the Restricted Isometry Property (RIP) [11] and incoherence with the basis matrix at which the sensed signal is sparse. These conditions on the sensing matrix are required for signal reconstruction. Signal reconstruction is a solution to an $l 1$-norm optimization problem to recover the high dimensional data from the low dimensional samples.

In previous work using CS with CRs, sensing a compressed measurements is done first, then reconstructing the Nyquist rate signal, and finally applying the spectrum sensing technique on the reconstructed signal or the reconstructed frequency response [12]-[14]. Although this method solves the high sampling rates problem, the resulting increase in computation and complexity is non-trivial. This results from the reconstruction step which is the most complex part in CS as will be discussed below.

In this paper we propose performing spectrum sensing from the compressed measurements sensed using CS directly without going into the intermediate process of reconstructing the signal. Therefore, hardware complexity will be reduced to a large extent. This can be realized by designing a compressive sensing process which guarantees that the information used in the detection process is preserved in the compressed measurements. To achieve this, we have to design a sensing matrix with some additional constraints differ from those used in the conventional CS [11]. In this paper we use the cyclostationary spectrum sensing technique on compressed measurements sensed by the Discrete Fourier Transform (DFT) sensing matrix. Cyclostationary feature detection has an advantage of its robustness against noise uncertainties which makes it one of the most powerful spectrum sensing techniques. Using the DFT sensing matrix enables us to make detection from the compressed measurements as will be discussed below. The results show that the 
performance is better than when using the traditional cyclostationary detection at the Nyquist-rate. This is in addition to the complexity reduction due to canceling the reconstruction step and solving the high sampling rates problem.

The remainder of the paper is organized as follows. In Section 2.1, we investigate some cyclostationary signal analysis techniques. System model and the traditional cyclostationary detector are mentioned in Section 2.2. Compressive sensing based cyclostationary detection (CSBCD), and the compressed measurements based cyclostationary detection (CMBCD) algorithms are discussed in Sections 2.3 and 2.4 respectively. Section 3 includes the simulation results and its discussion. Finally, conclusions are drawn in Section 4.

\section{System model and detection algorithms}

\subsection{Cyclostationary signal analysis}

Modulated signals have a built-in periodicity due to their coupling with periodic sinusoidal carriers, repeating spreading, over sampling or pulse trains. A random process $x(t)$ is classified as a wide sense cyclostationary process if the mean and autocorrelation are periodic in time, i.e.,

$$
\begin{aligned}
& E_{x}(t)=E_{x}\left(t+m T_{0}\right), \\
& R_{x}(t, \tau)=E\{x(t+\tau / 2) x(t-\tau / 2)\}=R_{x}\left(t+m T_{0}, \tau\right)
\end{aligned}
$$

where $\tau$ is the time lag, $T_{0}$ is the period and $m$ is an integer. Since $R_{x}(t, \tau)$ is periodic, it can be expressed as a Fourier series representation [15]

$$
R_{x}(t, \tau)=\sum_{\alpha=-\infty}^{\infty} R_{x}^{\alpha}(\tau) e^{j 2 \pi \alpha t}
$$

and

$$
R_{x}^{\alpha}(\tau)=\lim _{\Delta t \rightarrow \infty} \frac{1}{\Delta t} \int_{-\Delta t / 2}^{\Delta t / 2} x(t+\tau / 2) x(t-\tau / 2) e^{-j 2 \pi \alpha t} d t
$$

where $\alpha$ is the cyclic frequency and $\Delta t$ is the measurement interval.

Using the Wiener relationship, we can define the spectrum correlation function (SCF) as

$$
S_{x}^{\alpha}(f)=\int_{-\infty}^{\infty} R_{x}^{\alpha}(\tau) e^{-j 2 \pi f \tau} d \tau .
$$

An alternative expression for SCF is given by [15]

$$
S_{x}^{\alpha}(f)=\lim _{\Delta f \rightarrow \infty} \lim _{\Delta t \rightarrow \infty} \frac{1}{\Delta t} \int_{-\Delta t / 2}^{\Delta t / 2} \Delta f X_{1 / \Delta f}\left(t, f+\frac{\alpha}{2}\right) \tilde{X}_{1 / \Delta f}\left(t, f-\frac{\alpha}{2}\right) d t
$$

where

$$
X_{1 / \Delta f}(t, v)=\int_{t-1 / 2 \Delta f}^{t+1 / 2 \Delta f} x(u) e^{-j 2 \pi v u} d u
$$

which is the complex envelope of the narrow-band-pass component of $x(t)$; the received signal, with center frequency $v$ and bandwidth $\Delta f$.

Additive white Gaussian noise (AWGN) exhibits no cyclic correlation. Hence, the SCF 
of noise has no spectral features at $\alpha \neq 0$. Modulated signals have spectral features at $\alpha \neq 0$. For BPSK signals with carrier frequency $f_{c}$ and symbol rate $1 / T_{0}$, the SCF has peaks at $\alpha= \pm m / T_{0}, f= \pm f_{c}$ and $\alpha= \pm 2 f_{c} \pm m / T_{0}, f=0$ [16].

\subsection{System model and traditional cyclostationary detector}

In CRs the hypothesis model of the received signal is

$$
\begin{aligned}
& H_{0}: x(t)=n(t), \\
& H_{1}: x(t)=s(t)+n(t)
\end{aligned}
$$

where $x(t)$ is the received signal, $s(t)$ is the PU signal, $n(t)$ is AWGN, $H_{0}$ means that no primary user exists, and $H_{1}$ means that there is a primary user signal in this band.

Using the cyclostationary features for performing spectrum sensing, we can rewrite the hypothesis model considering the SCF as

$$
\begin{aligned}
& H_{0}: S_{x}^{\alpha}(f)=S_{n}^{\alpha}(f), \\
& H_{1}: S_{x}^{\alpha}(f)=S_{s}^{\alpha}(f)+S_{n}^{\alpha}(f)
\end{aligned}
$$

where $S_{n}^{\alpha}(f)$ is the SCF of noise, and $S_{s}^{\alpha}(f)$ is the SCF of the PU signal.

Because $n(t)$ is not a cyclostationary process, the SCF of it has no peaks at $\alpha \neq 0$. Therefore, the statistic test of the CD in the discrete domain is based on [17]:

$$
\xi=\sum_{\alpha, \alpha \neq 0} \sum_{f} S_{x}^{\alpha}(f) \tilde{S}_{x}^{\alpha}(f)
$$

where $\tilde{S}_{x}^{\alpha}(f)$ is the conjugate of $S_{x}^{\alpha}(f)$ which is the SCF of the received signal. Then this statistic value is compared with a predetermined threshold $\lambda$ to decide for $D_{0}$ or $D_{1}$

$$
\xi_{\substack{D_{1} \\ D_{1}}}^{D_{0}} \lambda
$$

where the result of test $D_{0}$ indicates receiving $H_{0}$, and $D_{1}$ indicates receiving $H_{1}$ after applying the detection algorithm.

\subsection{Compressive sensing based cyclostationary detection (CSBCD) algorithm}

In CSBCD, the received signal is sensed by CS to get a low dimensional data vector $\mathbf{y}$ using a sensing matrix $\Phi$ as follows

$$
\mathbf{y}=\boldsymbol{\Phi} \mathbf{x}
$$

where $\mathbf{X}$ is an $N \times 1$ vector which represents the Nyquist rate samples of $x(t), \mathbf{y}$ is 
the $M \times 1$ compressed measurements vector, and $\boldsymbol{\Phi}$ is an $M \times N$ sensing matrix $(M<<N)$.

After that, the Nyquist rate signal is reconstructed from the compressed measurements vector $\mathbf{y}$, and then the $C D$ is performed on the reconstructed signal. The reconstruction process is a linear inverse problem with sparseness constraint. It was shown that this problem is an NP-hard [18]. Basis Pursuit (BP) [19] is a reconstruction technique that transforms the problem to a convex optimization problem that can be solved by linear programming as follows

$$
\hat{\mathbf{x}}=\arg \min _{\mathbf{x}}\|\mathbf{x}\|_{1} \text { s.t. } \mathbf{y}=\boldsymbol{\Phi} \mathbf{x} .
$$

Several reconstruction techniques have been proposed in the literature. Examples of these techniques are Matching Pursuit (MP) [20] and Orthogonal Matching Pursuit (OMP) [21]. These techniques differ in the computational complexity and memory requirements. For instance, the computational complexity and memory requirements of the (OMP) algorithm are summarized in Table 1 where $k$ is the iteration number and $K$ is the final iteration number [22]. The accuracy of the reconstructed signal depends on the number of compressed measurements $M$ and the number of iterations.

\section{Table 1.}

Complexities and memory requirements for some OMP computational Approaches [22].

\begin{tabular}{|l|l|l|}
\hline Algorithm & Complexity & Memory \\
\hline Naive Way & $N M+M k+M k^{2}+k^{3}$ & $M N$ \\
\hline Cholesky Decomposition-1 & $N M+M k+k^{2}$ & $N^{2}+N M+k+k^{2}$ \\
\hline Cholesky Decomposition-2 & $N k+k^{2}$ & $N^{2}+N M+k+k^{2}$ \\
\hline QR Decomposition-1 & $N M+M k ;$ solve: $K^{2}$ & $N M+M k+k^{2}$ \\
\hline QR Decomposition-2 & $N k+M k+k^{2}$ & $N^{2}+N M+M k+k^{2}$ \\
\hline Matrix Inversion Lemma & $N k+M k$ & $N^{2}+N M+M k$ \\
\hline
\end{tabular}

2.4 Compressed measurements based cyclostationary detection (CMBCD) algorithm

Figure 1 shows the block diagram of the proposed algorithm. In this algorithm compressed measurements are sensed using a DFT sensing matrix as follows

$$
\Phi(k, i)=e^{-j \frac{2 \pi}{N} k i}
$$

where $\Phi$ is an $M \times N$ sensing matrix $(M<<N), i=0,1,2, \ldots, N-1$, and $k$ is the index of rows which represents the $M$ DFT coefficients taken out of $N$ coefficients. These indices are selected such that most of energy of $x$ is preserved in the compressed measurements $y$. For example, for a BPSK PU signal with main carrier frequency $f_{c}$ and sampling frequency $f_{s}$, most of the energy is concentrated around $k= \pm\left(f_{c} / f_{s}\right) N$. Therefore the $M$ coefficients are selected around these indices. 


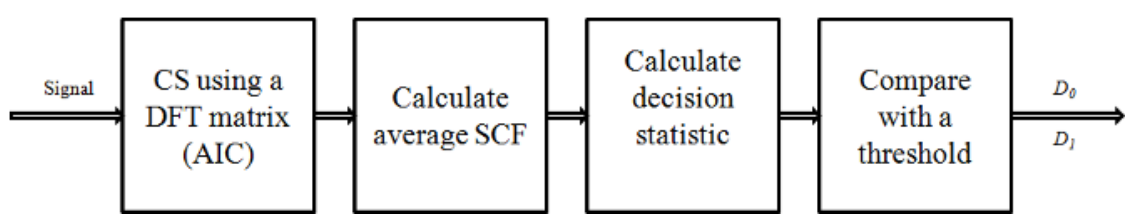

Fig. 1. System block diagram

After that, SCF is calculated by correlating the compressed measurements with itself and averaging over multiple frames as follows

$$
\begin{aligned}
& S_{x_{i}}^{\alpha}(f)=y(f+\alpha / 2) \tilde{y}(f-\alpha / 2), \\
& S_{x}^{\alpha}(f)=\frac{1}{n_{f}} \sum_{i=1}^{n_{f}} S_{x_{i}}^{\alpha}(f)
\end{aligned}
$$

where $S_{x_{i}}^{\alpha}(f)$ is the SCF for the $i^{\text {th }}$ frame, $\tilde{y}(f-\alpha / 2)$ is the conjugate of $y(f-\alpha / 2)$, and $n_{f}$ is the total number of frames used for averaging. To use another window we can multiply the columns of the sensing matrix by the window.

Finally calculate the detection statistic $\xi$ as in equation (12) and compare it with the threshold $\lambda$ to take the decision as in equation (13).

Performance of the detection algorithm can be measured with two probabilities: the probability of false alarm $P_{F}$, and the probability of detection $P_{D} . P_{F}$ is the probability that the hypothesis test incorrectly decides $H_{1}$ while it is $H_{0}$, and $P_{D}$ is the probability of correctly deciding $H_{1}$ while it is $H_{1}$, i.e.,

$$
\begin{aligned}
& P_{F}=\operatorname{Pr}\left(\xi>\lambda \mid H_{0}\right), \\
& P_{D}=\operatorname{Pr}\left(\xi>\lambda \mid H_{1}\right) .
\end{aligned}
$$

The probability of miss detection $P_{M}$ is given by

$$
\begin{aligned}
P_{M} & =1-P_{D}, \\
& =1-\operatorname{Pr}\left(\xi>\lambda \mid H_{1}\right) .
\end{aligned}
$$

\section{Numerical results and discussions}

Simulations were performed on a BPSK signal with the following parameters: main carrier frequency $f_{c}=125 \mathrm{MHz}$, bandwidth $20 \mathrm{MHz}$, and a sampling frequency $f_{s}=1$ GHz. The used window is a Hanning window. 


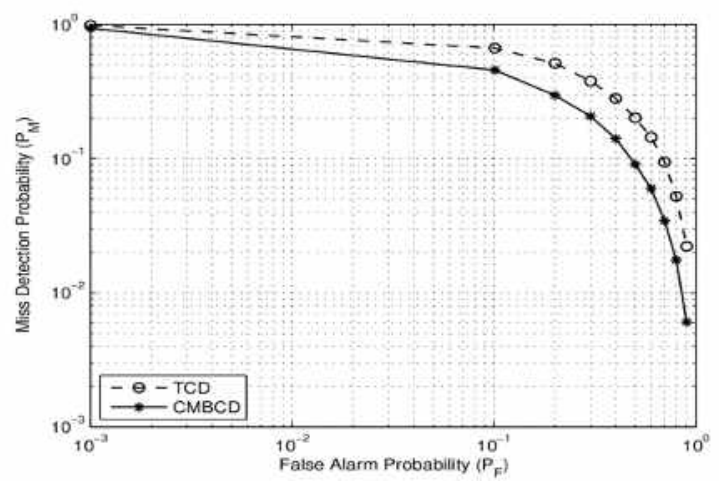

Fig. 2. Complementary ROC.

Detection of this signal was performed using the CMBCD algorithm and the traditional cyclostationary detector (TCD) method which uses the Nyquist rate signal. By each method, detection probabilities were calculated for different false alarm probabilities to plot the Receiver Operating Characteristics (ROC) curves. Figure 2 shows the ROC using the two methods, the TCD and the CMBCD algorithm with a compression ratio of 30\%, total number of samples of 1000 , window size of 200 , and signal to noise ratio (SNR) of -10 $\mathrm{dB}$. Although we used only $30 \%$ of the signal samples in CMBCD, performance is better than using all samples in the TED. This is because the compressed measurements contain most of the PU signal energy and only a fraction of the noise energy. Figure 3 shows the percentage of the energy contained in $M$ compressed measurements of both PU and noise signals against the compression ratio $(M / N)$. We find that, at $30 \%$ compression ratio, the percentage of the energy contained in the compressed measurements of the PU signal equals $97.77 \%$ and that of the noise signal equals $30 \%$. Therefore detection probability will increase relative to that of the TCD algorithm at the same false alarm probability.

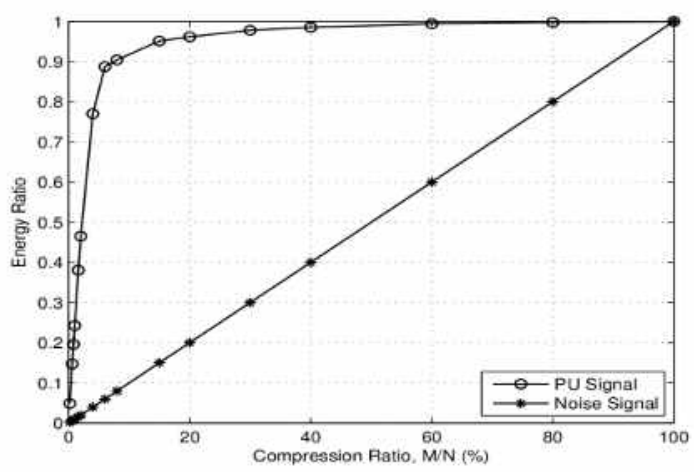

Fig. 3. Energy ratio versus compression ratio for AWGN and BPSK signal. 


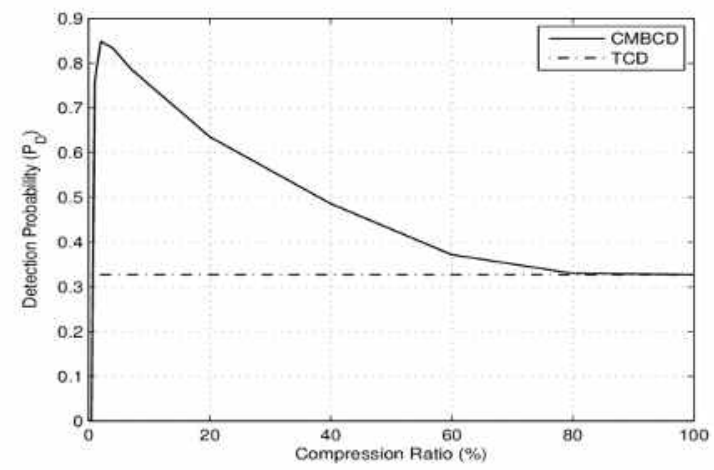

Fig. 4. Probability of detection vs. the compression ratio when $P_{F}=0.1$.

In the proposed algorithm, compression ratio can be decreased to a far extent, achieving a higher performance improvement. However, there is a limit in compression ratio as shown in Figure 4 which shows the probability of detection versus compression ratio at false alarm probability $P_{F}=0.1, \mathrm{SNR}=-10 \mathrm{~dB}$, and total number of sensed samples of 1000 .

Figure 5 shows the probability of detection versus signal to noise ratio at false alarm probability $P_{F}=0.1$, total number of sensed samples of 1000 , and a compression ratio of $30 \%$. We find that the detection probability of the proposed CMBCD algorithm is higher than that of the TED especially at low SNR regime. Figure 5: Probability of detection vs. SNR when $P_{F}=0.1$ and a compression ratio of $30 \%$.

\section{Conclusions}

In this paper, we proposed a spectrum sensing technique for wideband cognitive radio systems. In order for the SU to detect the existence of the PU, it first samples the received signal at a sub-Nyquist rate using compressive sensing and then applies the cyclostationary detection on the compressed measurements. Therefore, the complexity required in reconstructing the Nyquist rate signal is eliminated. In addition to the considerable complexity reduction, the results show that the proposed algorithm provides a better detection probability compared to traditional schemes at the same false alarm probability.

\section{REFERENCES}

[1] B. Wang and K. J. Ray Liu, "Advances in Cognitive Radio Networks: A Survey," IEEE Journal of Selected Topics in Signal Processing, vol. 5, no. 1, pp. 5-23, Feb. 2011.

[2] I. F. Akyildiz, W.-Y. Lee, M. C. Vuran, and S. Mohanty, "Next generation/dynamic spectrum access/cognitive radio wireless networks: A survey," Comput. Netw., vol. 50, pp. 2127-2159, May 2006.

[3] N. Yadav and S. Rathi, "Spectrum Sensing Techniques: Research, Challenge and Limitations," International Journal of Electronics \& Communication Technology (IJECT), vol. 2, issue 4, pp. 240-245, Oct.-Dec. 2011.

[4] M. Subhedar and G. Birajdar, "Spectrum Sensing Techniques in Cognitive Radio Networks: A Survey," International Journal of Next-Generation Networks (IJNGN), vol. 3, no. 2, pp. 37-51, June 2011.

[5] J. G. Proakis, M. Salehi, Digital Communications, 5th ed., Singapore:McGraw-Hill, 2008.

[6] F. F. Digham, M-S. Alouni and M. K. Simon, "On the Energy Detection of Unknown Signals 
Over Fading Channels,” IEEE Trans. Commun., vol 55, no.1, pp.21-24, Jan. 2007.

[7] I. F. Akyildiz, B. F. Lo, R. Balakrishnan, "Cooperative spectrum sensing in cognitive radio networks: A survey," Physical Communication, vol. 4, no. 1, pp. 40-62, 2011.

[8] Federal Communications Commission, "Notice of proposed rule making and order: Facilitating opportunities for flexible, efficient, and reliable spectrum use employing cognitive radio technologies," ET Docket No. 03-108, Feb. 2005.

[9] D. L. Donoho, “A Compressed Sensing," IEEE Trans. on Information Theory, vol. 52, pp. 1289-1306, April 2006.

[10] J. Haupt and R. Nowak, "Compressive Sampling for Signal Detection," Proc. IEEE Int. Conf. on Acoustics Speech and Signal Processing (ICASSP), vol. 3, pp. III-1509, April 2007.

[11] E. Candes, J. Romberg, and T. Tao, "Robust uncertainty principles: Exact signal reconstruction from highly incomplete frequency information," IEEE Trans. Inform. Theory, vol. 52, no. 2, pp. 489-509, Feb. 2006.

[12] Z. Tian, G. Giannakis, "Compressed Sensing for Wideband Cognitive Radios," International Conference on Acoustics, Speech, and Signal Processing (ICASSP),2007, Honolulu, Hawaii, vol. 4, pp. IV-1357, April 2007.

[13] Z. Tian, "Cyclic feature based wideband spectrum sensing using compressive sampling," Proc. IEEE Int. Conf. Commun. (ICC), pp. 1-5, June 2011.

[14] J.-A. Bazerque and G. B. Giannakis, "Distributed spectrum sensing for cognitive radio networks by exploiting sparsity," IEEE Trans. on Signal Process., vol. 58, no. 3, pp. 1847-1862, March 2010.

[15] Paul D. Sutton, Keith E. Nolan, and Linda E. Doyle, "Cyclostationary signatures in practical cognitive radio applications," IEEE Journal on Selected Areas in Communications, vol. 26, no. 1, pp. 13-24, 2008.

[16] Shiyu Xu, Zhijin Zhao, and Junna Shang, "Spectrum sensing based on cyclostationarity," IEEE Workshop on Power Electronics and Intelligent Transportation System, PEITS'08, pp. 171-174, August 2008.

[17] K. Sithamparanathan, A. Giorgetti, Cognitive Radio Techniques: Spectrum Sensing, Interference Mitigation, and Localization, Artech House, 2012.

[18] E. Candes, M. Rudelson, T. Tao and R. Vershynin, "Error correction via linear programming," Foundations of Computer Science, 2005 (FOCS 2005), 46th Annual IEEE Symposium on, pp. 668-681, 2005.

[19] S. S. Chen, D. L. Donoho, and M. A. Saunders, "Atomic decomposition by basis pursuit," SIAM J. Sci. Comput., vol. 20, no. 1, pp. 33-61, 1999.

[20] S. Mallat and Z. Zhang, "Matching pursuits with time-frequency dictionaries,"IEEE Transactions on Signal Processing, vol. 41, no. 12, pp. 3397-3415, 1993.

[21] J. Tropp and A. Gilbert, "Signal recovery from random measurements via orthogonal matching pursuit," IEEE Transactions on Information Theory, vol. 53, no. 12, pp. 4655-4666, 2007.

[22] B. L. Sturm, M. G. Christensen, "Comparison of orthogonal matching pursuit implementations," Signal Processing Conference (EUSIPCO), 2012 Proceedings of the 20th European, pp. 220-224, 27-31, August 2012. 


\section{مكتشف ثبات دورى قائم على القياسات المضغوطة لأنظمة الراديو المعرفى}

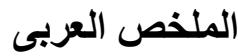

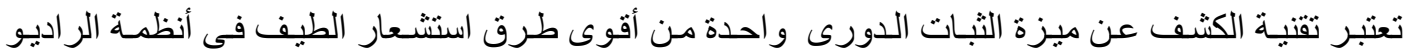

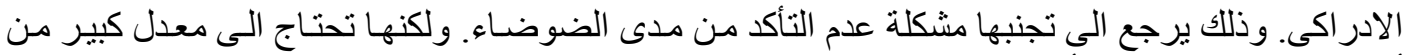

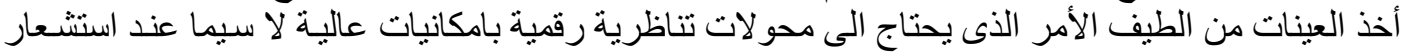

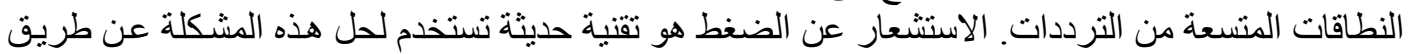

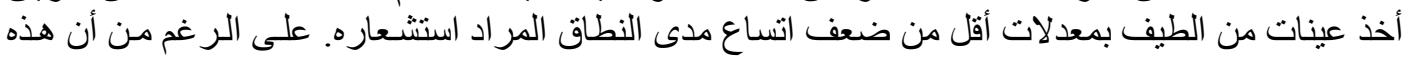

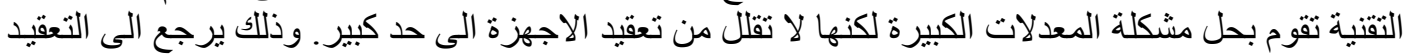

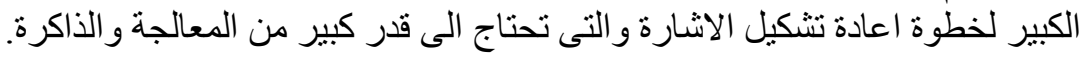

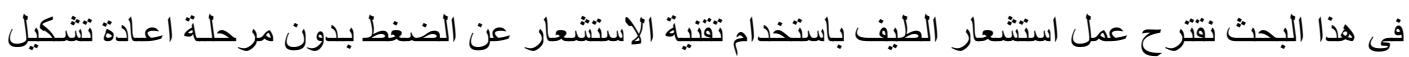

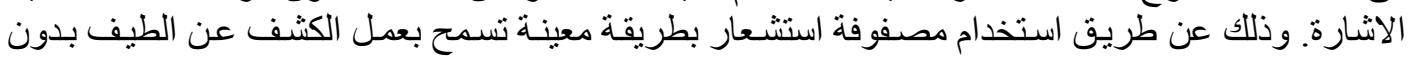

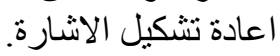

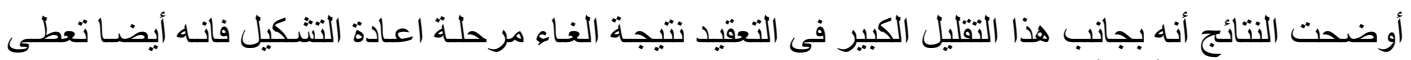

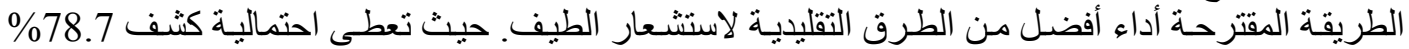
باستخدام عدد من العينات 7\% من العدد الذى تستخدمه الطرقة التقليدية و التى تعطى احتمالية كثف كنف 32.7\% 